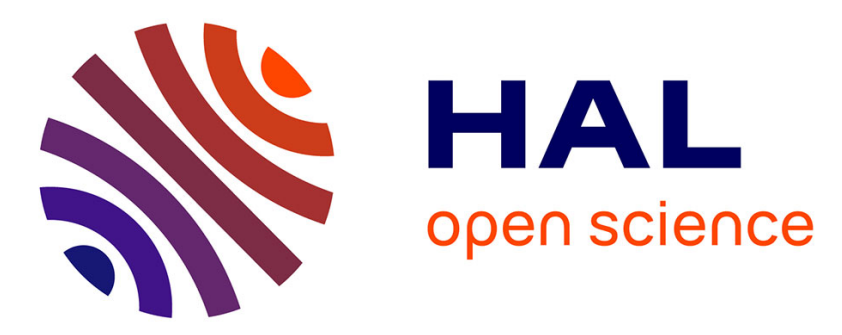

\title{
Les habitats forestiers de la France tempérée: typologie et caractérisation phytoécologique
}

\author{
Benoît Renaux, Jean-Claude Gégout
}

\section{To cite this version:}

Benoît Renaux, Jean-Claude Gégout. Les habitats forestiers de la France tempérée: typologie et caractérisation phytoécologique. Revue forestière française, 2010, 62 (3-4), pp.365-374. 10.4267/2042/38949 . hal-03449703

\section{HAL Id: hal-03449703 https://hal.science/hal-03449703}

Submitted on 25 Nov 2021

HAL is a multi-disciplinary open access archive for the deposit and dissemination of scientific research documents, whether they are published or not. The documents may come from teaching and research institutions in France or abroad, or from public or private research centers.
L'archive ouverte pluridisciplinaire HAL, est destinée au dépôt et à la diffusion de documents scientifiques de niveau recherche, publiés ou non, émanant des établissements d'enseignement et de recherche français ou étrangers, des laboratoires publics ou privés. 


\title{
LES HABITATS FORESTIERS DE LA FRANCE TEMPÉRÉE : TYPOLOGIE ET CARACTÉRISATION PHYTOÉCOLOGIQUE
}

\author{
Benoît Renaux - Jean-Claude Gégout
}

La traduction et l'adaptation à la France de la typologie européenne des habitats naturels CORINE biotopes ont constitué une première structuration à l'échelle nationale des écosystèmes français en "Habitats". Cette typologie identifie plusieurs dizaines d'unités forestières décrites en quelques lignes par leur type de végétation, leur localisation géographique et leurs conditions écologiques générales. La description floristique et écologique des unités reste cependant partielle et leur identification sur le terrain délicate à réaliser. La directive européenne 92/43 concernant la conservation des habitats naturels (dite directive " Habitats ") fournit une liste des habitats à forte valeur patrimoniale et dont la conservation nécessite la désignation de zones spéciales de conservation. Ces habitats sont définis à partir de la composition floristique des communautés végétales selon la classification phytosociologique. Les Cahiers d'habitats (Bensettiti et al., 2001) et le document Gestion forestière et diversité biologique - Identification et gestion intégrée des habitats et espèces d'intérêt communautaire (Rameau et al., 2000) décrivent les habitats de la directive présents sur le territoire français. Chaque habitat y est caractérisé, de façon plus fine que dans la typologie CORINE biotopes, à partir de l'expertise des auteurs et de la bibliographie, dans une fiche synthétique décrivant la flore présente et les conditions écologiques générales.

L'absence d'informations concernant les habitats non décrits dans la directive, associée au besoin d'étayer les descriptions des experts par des données floristiques et écologiques quantitatives, sont à l'origine de la réalisation de ce nouveau document sur les habitats forestiers français. Il propose pour la première fois une caractérisation précise et quantitative des conditions floristiques, climatiques et édaphiques des habitats forestiers de la France tempérée, hors zone méditerranéenne, qu'ils soient inclus ou non dans la directive " Habitats ». La typologie des habitats décrits est basée sur le niveau de l'association de la classification phytosociologique.

\section{CADRE TYPOLOGIQUE}

Approche intégratrice des conditions du milieu, la phytosociologie a pris une place importante dans l'édification de la typologie CORINE biotopes et dans l'identification des habitats d'intérêt communautaire. De nombreuses études phytosociologiques régionales ont par ailleurs proposé des référentiels à l'échelle de la zone étudiée, bien adaptés à une utilisation locale. À l'échelle nationale, elles se révèlent d'utilisation délicate du fait de la multiplication des syntaxons souvent redondants entre plusieurs études, créant des problèmes de synonymie ou d'homonymie. Fruit d'une large collaboration entre les phytosociologues français, le Prodrome des végétations de France (Bardat et al., 2004) propose une synthèse phytosociologique nationale. II ne fournit cependant pas de description des unités, ni de liste d'espèces, ce qui rend son utilisation peu aisée par le néophyte. En outre, il ne décrit les communautés végétales que jusqu'au rang de la 
sous-alliance, équivalent phytosociologique du genre en botanique, ce qui ne permet souvent pas de distinguer les habitats relevant de la directive " Habitats ».

Les typologies des stations forestières représentent une autre approche importante de classification des écosystèmes forestiers français en vue de leur gestion. Mise en œuvre en France depuis une trentaine d'années, cette démarche a conduit à la réalisation de plus d'une centaine de catalogues de types de stations forestières répartis sur le territoire national et décrivant chacun les écosystèmes forestiers d'une région naturelle. Les types de stations sont définis à partir du caractère bioindicateur de la flore spontanée et des conditions écologiques des stations de façon à regrouper dans un même type les écosystèmes aux potentialités forestières semblables. Des essences forestières à favoriser ont été associées, dans la plupart des cas, aux types de stations décrits. Les gestionnaires forestiers, publics ou privés, peuvent ainsi choisir de façon raisonnée les essences écologiquement les mieux adaptées aux conditions de milieu, et parfois adapter leurs itinéraires sylvicoles. Cependant, l'absence de couverture exhaustive de la France, le grand nombre de types de stations décrits (plus de 4000 pour la partie du territoire couverte par des catalogues), l'absence de correspondances entre types de stations de régions voisines et le caractère qualitatif des descriptions des types de stations, constituent autant d'obstacles pour une classification fine et homogène des écosystèmes forestiers à l'échelle nationale.

Qu'elles soient nationales ou régionales, les typologies actuelles ne permettent pas de traiter tous les habitats forestiers français de manière précise et quantitative et aucune typologie unifiée n'existe pour traiter des forêts françaises à un niveau de précision suffisant, le rang de l'association, utile au gestionnaire des milieux naturels. Dans ce cadre, l'ambition de ce travail est de proposer une typologie exhaustive des habitats forestiers français, hors zone méditerranéenne, tout en s'appuyant sur les ouvrages nationaux reconnus et abondamment utilisés que sont le Prodrome des végétations de France et les Cahiers d'habitats.

\section{VERS UNE TYPOLOGIE PHYTOSOCIOLOGIQUE NATIONALE POUR LES HABITATS FORESTIERS}

Le choix d'une typologie basée sur le niveau de l'association de la classification phytosociologique, définissant les habitats par l'approche des communautés végétales, s'est imposé dès le début. La phytosociologie possède l'avantage d'être reconnue et utilisée depuis des décennies au niveau européen, notamment dans la définition des habitats d'intérêt communautaire et la typologie CORINE biotopes. De plus, si l'utilisation de relevés phytoécologiques rend de prime abord possible la construction d'une typologie avec les outils statistiques modernes d'ordination et de classification, il est apparu pertinent de tirer parti de l'expérience d'un siècle de travaux européens sur le classement de la végétation, de l'abondante littérature phytosociologique et de la connaissance diffusée utilisant ce système. Enfin, l'échantillon de milieux naturels présents dans les bases de données est a priori non exhaustif de toutes les conditions françaises et l'utilisation d'une classification automatique réalisée sur les relevés des bases de données aurait abouti à une typologie fermée et lacunaire.

La première étape du travail a donc consisté à réaliser un synopsis des forêts françaises, vaste synthèse à travers la littérature phytosociologique européenne, puis à clarifier synonymies et homonymies entre les syntaxons : en effet, deux noms différents trouvés dans deux études différentes peuvent être synonymes, et a contrario un même nom peut désigner deux groupements différents sous la plume de deux auteurs différents. Ce sont ainsi 600 références qui ont été exploitées, dont de nombreuses synthèses majeures, aboutissant à un synsystème rassemblant environ 230 associations. Cette première version du document était identique, pour les habitats 
de la directive, à celle retenue dans les Cahiers d'habitats et le document Gestion forestière et diversité biologique (même classification, mêmes noms en latin et français). Elle était complétée, pour les habitats ne relevant pas de la directive "Habitats ", de façon à fournir une présentation homogène de tous les habitats forestiers identifiés en France tempérée. Jusqu'au rang de la sous-alliance, elle était conforme au Prodrome des végétations de France.

Cette première version du document a été testée sur le terrain et soumise à l'avis d'un groupe d'experts issus d'organismes d'étude et de gestion des forêts, de gestionnaires et de naturalistes ${ }^{(1)}$.

Leurs remarques et avis ont été précieux pour améliorer les premières versions du document, dans une volonté de concertation visant à aboutir à un consensus le plus large possible autour d'une typologie commune des forêts françaises. Si le fond - contenu des fiches habitats posait peu de problèmes, l'essentiel des remarques concernait la nomenclature phytosociologique, cette discipline obéissant à des règles comparables à celles de la botanique pour définir les groupements végétaux conformément au code international de nomenclature phytosociologique (Weber et al., 2000).

La volonté de réaliser un document consensuel s'est traduite par plusieurs collaborations majeures : avec R. Bœuf de la Direction territoriale Alsace de l'Office national des forêts et auteur du référentiel des types forestiers d'Alsace (Bœuf, sous presse ; voir également article de Bœuf dans ce numéro), et J.-M. Royer, co-auteur du Synopsis commenté des groupements végétaux de la Bourgogne et de la Champagne-Ardenne (Royer et al., 2006). Notre typologie a ainsi été mise en concordance avec le référentiel alsacien et nous avons repris de nombreux éléments du second ouvrage (syntaxons nouveaux et validation d'associations). Ceci s'est également traduit par un travail commun sur les syntaxons, notamment ceux décrits par J.-C. Rameau et non publiés ( $c f$. l'article de Renaux, Bœuf et Royer dans ce numéro). Ces collaborations s'inscrivent enfin dans la déclinaison du Prodrome des végétations de France jusqu'à l'association pour les forêts, travail auquel la première version du référentiel des habitats a apporté une contribution majeure. En retour, ce travail collégial a permis d'améliorer notre document, puisqu'il reprend dans sa version actuelle les choix validés par le groupe de travail sur les Querco roboris-Fagetea sylvaticae Br.-Bl. et Vlieger in Vlieger 1937, les forêts feuillues et mixtes les plus répandues en France tempérée, de l'étage collinéen au montagnard.

\section{CHOIX ET TRAITEMENT DES DONNÉES POUR DÉCRIRE LES HABITATS FORESTIERS}

Dans le contexte actuel de changement climatique, il est nécessaire de disposer de données quantitatives précises sur les habitats, afin non seulement de les caractériser précisément aujourd'hui (facteurs thermiques, hydriques, trophiques, espèces caractéristiques, répartition...) mais aussi de prédire les menaces qui pèsent sur leur persistance à long terme. La caractérisation précise et quantitative des conditions floristiques, climatiques et édaphiques des habitats forestiers que propose le document est rendue possible par l'utilisation massive de relevés phytoécologiques. Ces relevés sont issus de deux bases de données : EcoPlant et Sophy. EcoPlant est la base de données d'AgroParisTech-ENGREF et rassemble 9 ooo relevés forestiers dont un grand nombre dispose de profils et d'analyses de sols en plus des données floristiques (Gégout, 2001 ; Gégout et al., 2005). Ces relevés incluent tous les exemples type de stations des catalogues de stations forestières réalisés en France mais aussi des données de thèses, des réseaux européen

(1) Conservatoires botaniques nationaux, Muséum national d'histoire naturelle, Office national des forêts, Institut pour le développement forestier, Société française de phytosociologie, Centres régionaux de la propriété forestière. 
Benoît Renaux - Jean-Claude Gégout

et RENECOFOR, et d'études phytosociologiques. SOPHY est la base floristique développée par le CNRS (Brisse et al., 1995) et compte plus de 150 ooo relevés, dont 32000 identifiés comme forestiers complétant ceux d'EcoPlant. Une partie de ces relevés a pu être rattachée à la typologie des habitats, en priorité ceux disposant d'analyse de sols $(6000)$ et ceux sans données pédologiques mais issus de travaux phytosociologiques majeurs, contenant entre autres les relevés types des syntaxons. Parmi les 12000 relevés examinés, environ un quart n'a pas pu être rattaché au référentiel du fait de sylvofaciès trop artificialisés (plantations monospécifiques, peuplements avec espèces exotiques) ou de relevés floristiques non typiques car trop pauvres en espèces ou faisant transition entre deux groupements.

Afin d'éviter les problèmes d'auto-corrélation spatiale dus à la répartition des relevés en agrégats correspondant à autant d'études régionales, les relevés ont été ré-échantillonnés : si plusieurs relevés étaient trop proches géographiquement, seuls ceux considérés comme écologiquement différents ont été conservés ${ }^{(2)}$. Cette règle n'a pas été suivie pour les relevés issus de la publication contenant le type nomenclatural de l'association phytosociologique pour laquelle tous les relevés ont été utilisés, de manière à prendre en compte complètement la définition historique et officielle de l'association.

Aux données mesurées sur le terrain (profils et analyses de sol, topographie, altitude, cortège floristique...) ont été ajoutées des données climatiques ainsi que des valeurs bio-indiquées de paramètres nutritionnels et hydriques des sols, ce qui constitue une avancée importante dans la description des habitats forestiers français. Les données climatiques ont été calculées pour chaque relevé grâce à ses coordonnées géographiques par croisement sous système d'information géographique avec des données spatialisées issues d'un modèle d'interpolation des données des postes météorologiques sur la période 1960-1990 (modèle AURELHY de Météo France). Cette opération permet de prendre en compte le climat régional mais aussi les variations locales de la topographie. Si l'utilisation de données pédologiques tire largement parti des catalogues de stations, leur synthèse pour chaque habitat constitue une nouveauté. Les variables pédologiques mesurées en laboratoire ont été complétées par les valeurs bio-indiquées par la flore de ces variables pour les relevés ne disposant pas d'analyses de sol. Le calcul du caractère bioindicateur est réalisé grâce aux relevés phytoécologiques d'EcoPlant disposant de données mesurées. Une courbe de réponse des espèces a pu être modélisée pour chaque variable étudiée. Cette courbe, qui fournit l'évolution de la probabilité de présence de l'espèce le long du gradient étudié, permet de déterminer une valeur indicatrice qui correspond au maximum de probabilité de présence de l'espèce (son préférendum) pour le facteur. Il est ensuite possible de bio-indiquer les facteurs pour un relevé donné en moyennant les valeurs indicatrices des espèces présentes sur le site, mais aussi pour chaque habitat en moyennant les résultats obtenus pour chacun des relevés. Cette approche permet de disposer de données écologiques même pour les habitats qui n'ont que des relevés floristiques simples, comme c'est souvent le cas pour les relevés phytosociologiques.

\section{CONTENU DES FICHES DÉCRIVANT LES HABITATS FORESTIERS FRANÇAIS}

\section{Description des conditions écologiques}

Les descripteurs thermiques, trophiques et hydriques retenus pour caractériser les habitats ont été choisis pour leur caractère explicatif de la répartition de la flore forestière française, selon l'avis de différents experts et les résultats d'une analyse factorielle des correspondances (AFC)

(2) Habitat différent ou altitude différente d'au moins $50 \mathrm{~m}$ ou pH différent d'au moins une demi-unité ou exposition différente d'au moins $90^{\circ}$ ou rapport $\mathrm{C} / \mathrm{N}$ différent d'au moins une unité ou topographie, type d'humus, type de sol différents. 
réalisée sur 16427 relevés floristiques répartis sur toute la France. Pour les principaux descripteurs, les gammes de valeurs où l'habitat est présent (déciles 1,5 et 9 de la distribution des valeurs de la variable déterminées sur les relevés classés dans l'habitat) sont indiquées graphiquement (voir figure 1, ci-dessous). Cette gamme de valeur est positionnée dans l'ensemble des valeurs de la variable correspondant aux forêts en France (par exemple de o à $2500 \mathrm{~m}$ pour l'altitude, voir figure 1, ci-dessous).

Les données climatiques fournies caractérisent chaque habitat du point de vue de la température et du bilan hydrique. Les températures moyennes annuelles, de janvier et de juillet ainsi que les précipitations annuelles et divers indices de bilan hydrique (P - ETP de mai à septembre, indice de de Martonne annuel) sont indiqués pour chaque habitat. La gamme d'altitudes de présence de l'habitat est également mentionnée. L'exemple de trois habitats situés dans trois étages de végétation est particulièrement illustratif (figure 1, ci-dessous). Le document permet non seulement de bien différencier les habitats de climats collinéens, montagnards ou subalpins mais aussi de distinguer au sein des étages de végétation les habitats de milieux secs des autres habitats. Les pineraies de Pin à crochets à Ononide à feuilles rondes situés dans des conditions à précipitations très faibles (660 $\mathrm{mm}$ en moyenne) bien que situées en haute montagne, en sont un bon exemple (figure 1, ci-dessous).

FIGURE 1

GAMMES DE CONDITIONS CLIMATIQUES ET D'ALTITUDE pour trois habitats, subalpin, montagnard et collinéen, des forêts françaises

De haut en bas : les pineraies sèches de Pin à crochets à Ononide à feuilles rondes des Alpes internes (association Ononido rotundifoliae-Pinetum uncinatae Bartoli 1966, code CORINE biotopes : 42-4215, EUR27 : 9430), les sapinières-hêtraies à Dentaire pennée (association : Cardamino heptaphyllae-Abietetum albae (Moor 1952) Hartmann et Jahn 1967, code CORINE biotopes : 41-13, EUR27 : 9130), les chênaies sessiliflorescharmaies à Alisier blanc (association Sorbo ariae-Quercetum petraea (Rameau 1974, 1996) Renaux, Royer et Bœuf 2010, code CORINE biotopes : 41-2).

La gamme des conditions propre à chaque habitat est représentée par un trait gras joignant les $1^{\mathrm{er}}$ et $9^{\text {e }}$ déciles de la distribution de la variable dans les relevés de l'habitat. L'optimum de l'habitat, mentionné par un trait vertical, correspond au $5^{\mathrm{e}}$ décile de la distribution. L'ensemble est présenté dans la gamme de valeurs de la variable où sont trouvés les écosystèmes forestiers français.

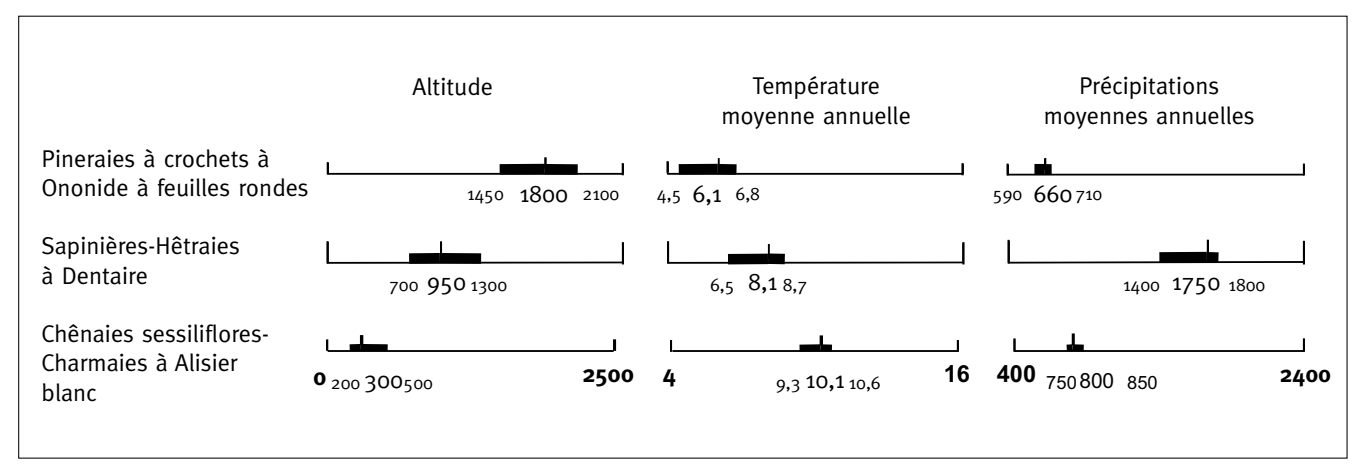

Les données édaphiques fournies pour chaque habitat concernent essentiellement l'acidité du sol $(\mathrm{pH}$ de l'horizon $\mathrm{A})$, la qualité de sa nutrition en potassium, calcium et magnésium et l'intensité de la toxicité aluminique (rapport $\mathrm{S} / \mathrm{T}=(\mathrm{Ca}+\mathrm{Mg}+\mathrm{K}) /(\mathrm{Ca}+\mathrm{Mg}+\mathrm{K}+\mathrm{Al}+\mathrm{H})$ de l'horizon $\mathrm{A}$ ), la qualité de sa nutrition azotée ( $\mathrm{C} / \mathrm{N}$ de l'horizon $\mathrm{A})$, la réserve utile en eau maximale et l'intensité de l'hydromorphie. La gamme de valeurs de présence de chaque habitat pour ces différents paramètres est présentée de façon identique à celle du climat (figure 2, p. 370). Ici aussi, la gamme des valeurs des paramètres édaphiques montre clairement une nette spécialisation de 
chaque habitat dans des conditions de sols particulières. Par exemple, les hêtraies-chênaies acidiphiles collinéennes continentales à Luzule blanchâtre sont généralement situées sur des sols de $\mathrm{pH}_{\text {eau }}$ de l'horizon $\mathrm{A}$ inférieur à 4,5, les hêtraies-chênaies " à Pâturin de chaix " sont sur des sols à $\mathrm{pH}$ faiblement acide compris entre 4 et 5 , alors que les hêtraies-chênaies à Aspérule odorante sont clairement situées dans des milieux neutres à calcaires à $\mathrm{pH}$ supérieur à 5,5 (figure 2, ci-dessous).

FIGURE 2

GAMME DE CONDITIONS NUTRITIONNELLES (pH ET C/N) de trois habitats de hêtraies-chênaies

De haut en bas : les hêtraies, hêtraies-chênaies acidiphiles collinéennes continentales à Luzule blanchâtre (association : Fago sylvaticae-Quercetum petraeae Tüxen 1955 race médioeuropéenne, code CORINE biotopes : 41-111, EUR27 : 9110), les hêtraies-chênaies " à Pâturin de Chaix " (association Deschampsio cespitosaeFagetum sylvaticae (Rameau 1996) Renaux, Bœuf et Royer, code CORINE biotopes 41-13, EUR27 : 9130), les hêtraies-chênaies à Aspérule odorante et Mélique uniflore (association : Carici flaccae-Fagetum sylvaticae Thill 1964 = Galio odorati-Fagetum sylvaticae Rübel 1930 ex. Sougnez et Thull 1959 p.p., code CORINE biotopes : 41-131, EUR27 : 9130). Le pH et le C/N sont des indicateurs d'acidité et de nutrition azotée des sols (les C/N faibles indiquent de bonnes conditions de nutrition azotée).

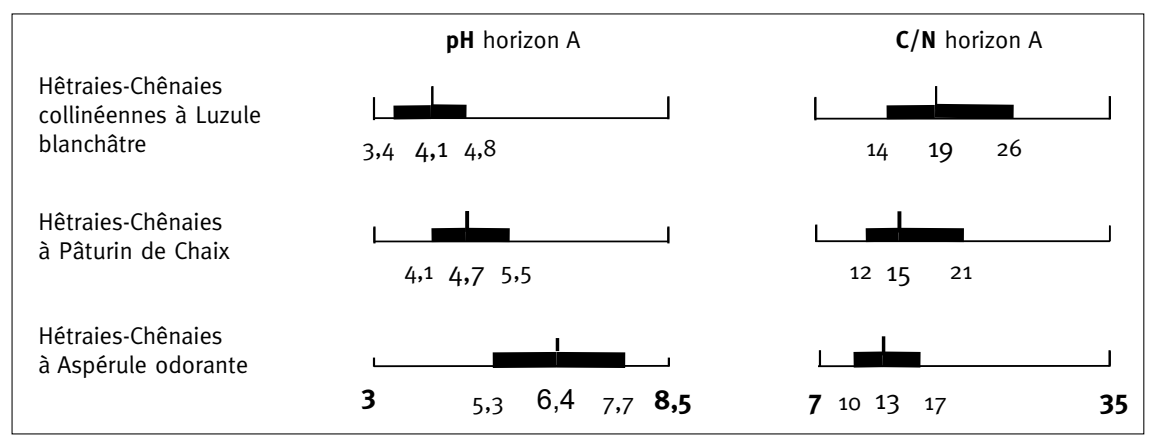

Lorsque le nombre de sites disposant d'analyses de sol était suffisant pour un habitat, divers autres paramètres édaphiques ont été fournis dans les fiches descriptives des habitats. Ils sont relatifs aux caractéristiques chimiques du sol $\left(\mathrm{Ca}, \mathrm{Mg}, \mathrm{H}\right.$, Al échangeables, $\mathrm{P}_{2} \mathrm{O}_{5}$ ) ainsi qu'à ses caractéristiques physiques (pierrosité, texture) et à son engorgement.

\section{Description de la flore des habitats}

Dans la description du cortège floristique d'un habitat, la fréquence des espèces est complétée par leur indice de fidélité pour cet habitat, ce qui apporte une information supplémentaire car une espèce peut être fréquente sans être caractéristique. Cet indice de fidélité calculé par la méthode de Chytrý et al. (2002) est d'autant plus élevé que l'espèce est typique de l'habitat. Sa valeur est calculée à partir du nombre de relevés dans le jeu de données et dans l'habitat et du nombre d'occurrences de l'espèce dans le jeu de données et dans l'habitat :

Formule de l'indice de fidélité (Chytrý et al., 2002)

$$
\Phi=\frac{N \times n_{p}-n \times N_{p}}{\sqrt{n \times N_{p} \times(N-n) \times\left(N-N_{p}\right)}}
$$

$\mathrm{N}=$ nombre de relevés dans le jeu de données

$\mathrm{n}=$ occurrence de l'espèce dans le jeu de données

$\mathrm{N}_{\mathrm{p}}=$ nombre de relevés dans l'habitat

$n_{p}=$ occurrence de l'espèce dans l'habitat 
Cet indice, indépendant de la taille du jeu de données, varie de 1 lorsque l'espèce est présente dans tous les relevés de l'association et absente dans tous les autres à - 1 lorsque l'espèce est absente de tous les relevés de l'association et présente dans tous les autres. Les valeurs positives indiquent une fréquence de l'espèce dans l'association plus grande qu'attendue par le hasard. Les valeurs proches de o indiquent que l'espèce est distribuée de façon aléatoire dans l'unité et le reste du jeu de données. Ainsi, les espèces possédant l'indice le plus élevé constituent une combinaison caractéristique de chaque habitat qui ne se substitue pas à celle donnée par l'auteur dans la publication historique, mais la complète. En effet, les phytosociologues ont

TABLEAU I Fréquence $(f)$ et indice de fidélité (If, ici valeur $=$ If $\times 10$ ) des 10 espèces les plus typiques de trois habitats fréquents dans le massif vosgien :

les hêtraies, hêtraies-sapinières acidiphiles de l'étage montagnard à Luzule blanchâtre (HS Luz., association : Luzulo luzuloidis-Fagetum sylvaticae Meusel 1937, code CORINE biotopes : 41-112, EUR27 : 9110), les sapinières-hêtraies vosgiennes à Fétuque des bois (SH Fet, assoc. Festuco altissimae-Abietetum albae Issler 1924 em. Hubert 1986, code CORINE biotopes : 41-13, EUR27 : 9130), les sapinières-hêtraies neutrophiles vosgiennes

à Mercuriale pérenne (SH Mer, assoc. Mercurialo perennis-Abietetum albae (Duchauffour 1954) Hubert 1986, code CORINE biotopes : 41-13, EUR27 : 9130). Ces trois habitats se trouvent sur des sols à $\mathrm{pH}_{\text {eau }}$ moyen de l'horizon A de 3,9, 4,2 et 5,0 respectivement.

\begin{tabular}{|c|c|c|c|c|c|c|}
\hline & \multicolumn{2}{|c|}{ HS Luz } & \multicolumn{2}{|c|}{ SH Fet } & \multicolumn{2}{|c|}{ SH Mer } \\
\hline & $f$ & IF & $f$ & If & $f$ & If \\
\hline Galium saxatile . . . . . . . . . . . . . . . . & 35 & 4,7 & & & & \\
\hline Deschampsia flexuosa .............. & 100 & 3 & & & & \\
\hline Plagiothecium undulatum. . . . . . . . . . & 13 & 2,7 & & & & \\
\hline Vaccinium myrtillus . . . . . . . . . . & 87 & 3,9 & 10 & & & \\
\hline Luzula sylvatica . . . . . . . . . . . . . & 61 & 3,7 & 22 & 1,9 & & \\
\hline Dryopteris dilatata. . . . . . . . . . . . & 70 & 3,4 & 99 & 8,7 & & \\
\hline Luzula luzuloides. . . . . . . . . . . . . . . . & 70 & 3,2 & 27 & 1,6 & & \\
\hline Picea abies . . . . . . . . . . . . . . . . . . . & 57 & 2,9 & 36 & 2,9 & & \\
\hline Sorbus aucuparia. . . . . . . . . . . . . . . . & 61 & 2,6 & 15 & 0,3 & 20 & 0 \\
\hline Rubus idaeus . . . . . . . . . . . . . . & 52 & 2,2 & 57 & 4,4 & 30 & 0 \\
\hline Abies alba. . . . . . . . . . . . . . & 83 & 3,6 & 88 & 6,7 & 70 & 1,9 \\
\hline Prenanthes purpurea............... & 26 & 1,1 & 52 & 4,6 & 30 & 0,1 \\
\hline Senecio ovatus . . . . . . . . . . . . . & 13 & 0,7 & 39 & 7,7 & 30 & 2,2 \\
\hline Festuca altissima . . . . . . . . . . & 17 & 0,6 & 93 & 9,6 & 60 & 2,2 \\
\hline Plagiomnium affine . . . . . . . . . . . & & & 25 & 5,9 & & \\
\hline Oxalis acetosella ............... & & & 76 & 4,7 & & \\
\hline Athyrium filix-femina . . . . . . . . . . . & & & 79 & 4,3 & 40 & 1,2 \\
\hline Stellaria nemorum . . . . . . . . . . . . . . & & & 36 & 4,2 & 30 & 0 \\
\hline Impatiens noli-tangere . . . . . . . . . . . & & & 40 & 3,7 & 60 & 2,2 \\
\hline Silene dioica . . . . . . . . . . . . . . . . . & & & 21 & 2,4 & 40 & 1,9 \\
\hline Mercurialis perennis. . . . . . . . . . . . . & & & 12 & 0 & 100 & 2,8 \\
\hline Cardamine heptaphylla. . . . . . . . . . & & & & & 40 & 1,9 \\
\hline Pulmonaria obscura............... & & & & & 30 & 2,2 \\
\hline Cardamine impatiens............... & & & & & 50 & 4,1 \\
\hline Lunaria rediviva. . . . . . . . . . . . & & & & & 60 & 4,4 \\
\hline
\end{tabular}


souvent défini les groupements dans une région restreinte ou par rapport aux syntaxons voisins. Les espèces diagnostiques fournies par les auteurs le sont donc par rapport aux forêts voisines et aux unités phytosociologiques proches. Au contraire, l'indice de fidélité est calculé dans le document habitats par rapport à 9000 relevés forestiers répartis dans toute la France et dans toutes les associations phytosociologiques. Le caractère diagnostique des espèces est donc valide à l'échelle du territoire national et par rapport à l'ensemble des associations décrites en France tempérée. L'exemple de trois habitats fréquents dans le massif vosgien, les hêtraies-sapinières à Luzule blanchâtre, à Fétuque des bois et à Mercuriale montre l'intérêt des deux paramètres pour caractériser les habitats : la fréquence des espèces donne une image de la flore des habitats alors que l'indice de fidélité en extrait les espèces typiques (tableau I, p. 371). Par rapport à l'approche phytosociologique traditionnelle, l'indice permet de hiérarchiser le caractère diagnostique des espèces. En plus des espèces typiques de chaque unité isolées également dans l'approche traditionnelle, l'approche nationale conduit à faire ressortir comme typiques des espèces communes aux trois unités, comme le Sapin, mais qui sont spécifiques des forêts de montagne par rapport à l'ensemble des syntaxons de plaine où ces espèces sont absentes.

\section{CONCLUSIONS}

L'ouvrage Les habitats forestiers de la France tempérée : typologie et caractérisation phytoécologique est destiné tant aux naturalistes et gestionnaires de milieux naturels qu'aux sylviculteurs. Il propose une description fine des facteurs physiques et biologiques de chaque habitat ainsi que sa valeur patrimoniale. Il permet aussi de saisir les caractéristiques et contraintes stationnelles influant sur la sylviculture et la croissance des essences : sensibilité des sols au tassement, engorgement, chimie du sol... Première synthèse exhaustive des habitats forestiers français, il se veut un pont entre plusieurs approches dans l'étude des végétations : la phytoécologie traduite notamment par les catalogues de stations forestières, la phytosociologie qui si elle se base sur l'étude des communautés végétales ne peut ignorer les facteurs écologiques, et enfin la notion d'habitat utilisée dans l'application de la directive européenne du même nom. L'utilisation de jeux de données nationaux de grande taille ainsi que de méthodes statistiques comme l'indice de fidélité de Chytrý apparaissent comme un apport significatif dans la pratique de la phytosociologie, qui ouvre la voie à un classement plus formalisé des milieux forestiers français. Disposer de relevés couplant données floristiques, climatiques et pédologiques ouvre également la voie à la modélisation de l'aire de répartition potentielle des habitats, dans le but de spatialiser leur répartition actuelle mais aussi future sous l'effet des changements climatiques. Ce document se veut enfin une contribution à l'édification d'une typologie nationale pour les végétations et notamment à la déclinaison du Prodrome des végétations de France au rang de l'association.
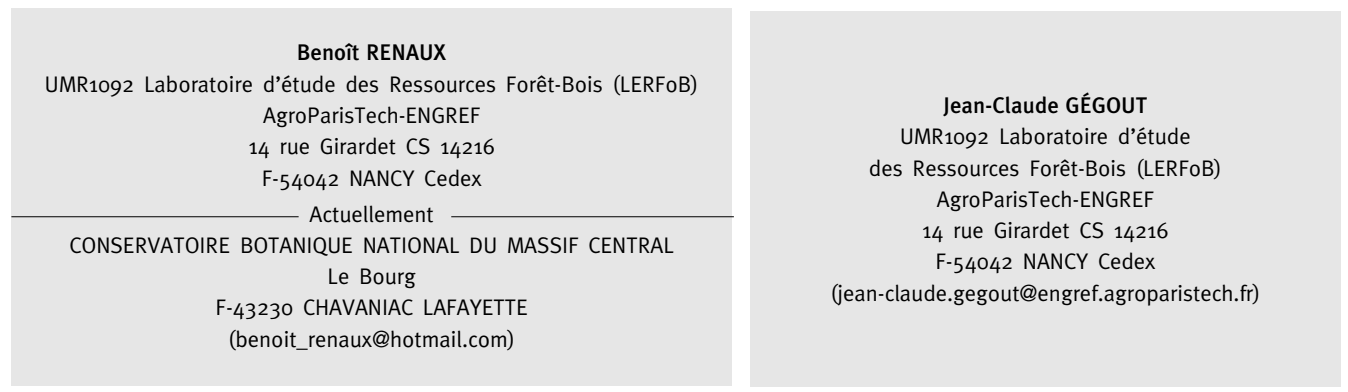


\section{Remerciements}

Nous exprimons nos remerciements à Richard Bœuf, Jean-Marie Royer et Damien Marage pour leur aide et leurs conseils dans le classement des relevés et la classification phytosociologique des syntaxons.

\section{BIBLIOGRAPHIE}

BRISSE (H.), RUFFRAY (P. de), GRANDJOUAN (G.), HOFF (M.). - European vegetation survey. The phytosociological database "Sophy". Part 1. Calibration of indicator plants. Part 2. Socio-ecological classification of the relevés. - Annali di botanica, vol. 53, 1995, pp. 177-223.

BARDAT (J.), BIORET (F.), BOTINEAU (M.), BOULLET (V.), DELPECH (R.), GÉHU (J.-M.), HAURY (J.), LACOSTE (A.), RAMEAU (J.-C.), ROYER (J.-M.), ROUX (G.), TOUFFET (J.). - Prodrome des végétations de France. Paris : Muséum national d'histoire naturelle, 2004. - 171 p. (Patrimoines naturels, vol. 61).

BENSETTITI (F.), RAMEAU (J.-C.), CHEVALLIER (H.) (coord.). - "Cahiers d'habitats » Natura 20oo. Connaissance et gestion des habitats et des espèces d'intérêt communautaire. Tome 1 - Habitats forestiers. MATE/MAP/MNHN. - Paris : La Documentation française, 2001. - 2 volumes : 339 p. et 423 p.

BFUF (R.). - Référentiel des types forestiers d'Alsace ; relations entre les stations forestières, les communautés forestières et les habitats. - Document ONF-SRFB. - Fontainebleau : Office national des Forêts. - 250 p. (sous presse).

CHYTRY (M.), TICHY (L.), HOLT (J.), BOTTA-DUKAT (Z.). - Determination of diagnostic species with statistical fidelity measures. - Journal of Vegetation Science, vol. 13, 2002, pp. 79-90.

GÉGOUT (J.-C.). - Création d'une base de données phytoécologiques pour déterminer l'autécologie des espèces de la flore forestière de France. - Revue forestière française, vol. LIII, n 3-4 spécial "Les 40 ans de l'Inventaire forestier national : utilisation et valorisation des données collectées”, 2001, pp. 397-403.

GÉGOUT (J.-C.), COUDUN (C.), BAILLY (G.), JABIOL (B.). - EcoPlant : a forest site database linking floristic data with soil and climate variables. - Journal of Vegetation Science, vol. 16, 2005, pp. 257-260.

GÉGOUT (J.-C.), RAMEAU (J.-C.), RENAUX (B.), JABIOL (B.), BAR (M.), MARAGE (D.). - Les habitats forestiers de la France tempérée ; typologie et caractérisation phytoécologique. - Nancy : AgroParisTech-ENGREF, 2008. - 720 p., 6 annexes.

RAMEAU (J.-C.), GAUBERVILLE (C.), DRAPIER (N.). - Gestion forestière et diversité biologique. Identification et gestion intégrée des habitats et espèces d'intérêt communautaire. France domaine continental. Paris : ENGREF-ONF-IDF, 2000.

ROYER (J.-M.), FELZINES (J.-C.), MISSET (C.), THÉVENIN (S.). - Synopsis commenté des groupements végétaux de la Bourgogne et de la Champagne-Ardenne. - Bulletin de la Société botanique du Centre-Ouest, numéro spécial 25, 2006, 394 p.

WEBER (H.E.), MORAVEC (J.), THEURILLAT (J.-P.). - International code of phytosociological nomenclature. $3^{\text {rd }}$ edition. - Journal of Vegetation Science, vol. 11, 2000, pp. 739-768. 


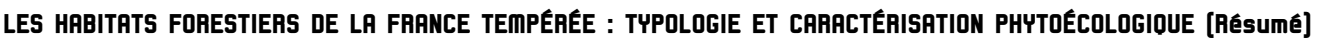

Fruit de l'exceptionnelle connaissance des écosystèmes forestiers de Jean-Claude Rameau et d'une vaste synthèse de la littérature phytosociologique européenne, le document Les habitats forestiers de la France tempérée - Typologie et caractérisation phytoécologique propose pour la première fois un synsystème décliné jusqu'au niveau de l'association pour les forêts de la France métropolitaine (hors zone méditerranéenne et Corse), qu'elles soient ou non concernées par la directive "Habitats". Encore en cours d'amélioration, il s'inscrit dans le prolongement des documents de référence en la matière (Cahiers d'habitats, Prodrome des végétations de France, document Gestion forestière et diversité biologique...) ; il se propose de les compléter, en lien étroit avec la déclinaison du Prodrome des végétations de France, travail dans lequel il s'inscrit pleinement afin d'aboutir à une typologie commune aux deux documents.

Ce document donne accès, pour la première fois à cette échelle, à une caractérisation précise et quantitative des conditions floristiques, climatiques et édaphiques de plus de 180 associations forestières réalisée à l'aide de 9000 relevés floristiques et phytoécologiques issus essentiellement de la base de données EcoPlant.

\section{THE FOREST HABITATS OF TEMPERATE FRANCE - TYPOLOGY AND PHYTOECOLOGICAL CHARACTERISATION [Abstract]}

“Les habitats forestiers de la France tempérée - Typologie et caractérisation phytoécologique” is a synopsis of Jean-Claude Rameau's outstanding knowledge of forest ecosystems and of European phytosociological literature and constitutes the first synsystem of French Metropolitan forests at the association level. It covers all forest types, except Corsican and Mediterranean ones, including ones that are not in the European "Habitats" directive. It is based on the leading existing typologies (Cahiers d'habitats, Prodrome des végétations de France, Gestion forestière et diversité biologique document, etc.) and supplements them. Our typology is brought into line with the new version of the "Prodrome des végétations de France" which is still in process and will describe French vegetation types very precisely.

Using 9000 phytoecological relevés (mainly from the EcoPlant database), our typology provides detailed quantitative information, for the first time at this scale, about flora, climate and soil moisture for more than 180 forest associations. 